\title{
Existence of Waves for a Nonlocal Reaction-Diffusion Equation
}

\author{
I. Demin * and V. Volpert \\ Institut Camille Jordan, University Lyon 1, UMR 5208 CNRS \\ 69622 Villeurbanne, France
}

\begin{abstract}
In this work we study a nonlocal reaction-diffusion equation arising in population dynamics. The integral term in the nonlinearity describes nonlocal stimulation of reproduction. We prove existence of travelling wave solutions by the Leray-Schauder method using topological degree for Fredholm and proper operators and special a priori estimates of solutions in weighted Hölder spaces.
\end{abstract}

Key words: integro-differential equation, travelling waves, Leray-Schauder method AMS subject classification: $35 \mathrm{~K} 57$

\section{Introduction}

In this work we study the integro-differential equation

$$
\frac{\partial u}{\partial t}=\frac{\partial^{2} u}{\partial x^{2}}+J(u) u(1-u)-\alpha u
$$

where

$$
J(u)=\int_{-\infty}^{\infty} \phi(x-y) u(y, t) d y,
$$

$\phi(x)$ is a non-negative function with a bounded support and $\int_{-\infty}^{\infty} \phi(x) d x=1$. If we replace $\phi$ by a $\delta$-function, then instead of (1.1) we obtain the reaction-diffusion equation

$$
\frac{\partial u}{\partial t}=\frac{\partial^{2} u}{\partial x^{2}}+u^{2}(1-u)-\alpha u
$$

\footnotetext{
*Corresponding author. E-mail: demin@math.univ-lyon1.fr
} 
In population dynamics it describes evolution of a population density. The reproduction term $u^{2}(1-u)$ is proportional to the square of the density (sexual reproduction) and to available resources $(1-u)$. The last term in the right-hand side describes mortality of the population. In some cases the interaction of the individuals in the population can be nonlocal. Some biological examples are considered in [11], [17], [18]. This can be for example plants that can distribute their pollen in some area around their location or biological cells which can send signalling molecules. This nonlocal interaction can influence the reproduction term resulting in the appearance of the factor $J(u)$. In this case, instead of the reaction-diffusion equation we should consider the integrodifferential equation (1.1).

Reaction-diffusion waves described by equation (1.2) are studied by Kanel [10], Fife and McLeod [6], and by other authors (see [13] and the references therein). If $0<\alpha<1 / 4$, then the nonlinearity

$$
F(u)=u^{2}(1-u)-\alpha u
$$

has three zeros:

$$
w_{+}=0, \quad w_{0}=\frac{1}{2}-\sqrt{\frac{1}{4}-\alpha}, \quad w_{-}=\frac{1}{2}+\sqrt{\frac{1}{4}-\alpha} .
$$

In this case equation (1.2) has a travelling wave solution, that is a solution of the form $u(x, t)=$ $w(x-c t)$, with the limits $w( \pm \infty)=w_{ \pm}$at infinity. It is unique up to translation in space, and globally stable.

In this work we study the existence of waves for equation (1.1). In the other words, we look for solutions of the problem

$$
\begin{gathered}
w^{\prime \prime}+c w^{\prime}+J(w) w(1-w)-\alpha w=0 . \\
\lim _{x \rightarrow \pm \infty} w(x)=w_{ \pm} .
\end{gathered}
$$

This particular equation represents, for example, cell communitation during the process of hematopoiesis (production of red blood cells). The main result of this work is stated in the following theorem.

Theorem 1. There exists a monotone travelling wave, that is a constant c and a twice continuously differentiable monotone function $w(x)$ satisfying (1.3), (1.4).

In the case of the scalar reaction-diffusion equation, the proof of the wave existence is simple. It is sufficient to reduce the equation

$$
w^{\prime \prime}+c w^{\prime}+F(w)=0
$$

to a system of first order equations and to prove the existence of a heteroclinic trajectory. For obvious reasons, this method is not applicable for the integro-differential equation. The proof becomes much more involved and requires a rather sophisticated mathematical construction. It is 
based on the Leray-Schauder method which implies the existence of a topological degree for the corresponding operators and a priori estimates of solutions.

Topological degree for elliptic operators in unbounded domains is constructed using the properties of Fredholm and proper operators with the zero index [13, 14, 15]. The same construction can be used for the nonlocal reaction-diffusion operators. We discuss this question in Section 2. We need to use here weighted spaces. Otherwise the degree may not be defined.

The method to obtain a priori estimates of solutions is similar to the method developed for monotone reaction-diffusion systems [15]. It is based on the maximum principle which is applicable for the equations under consideration.

We note that if the integral enters the nonlinearity in a different way, for example $u(1-J)$, as it is the case for the nonlocal Fisher equation [9], then the maximum principle is not applicable. In this case, the properties of the equation become quite different. It possesses an interesting nonlinear dynamics $[4,8]$ but the wave existence can be proved only in the case of functions $\phi$ with a small support where the perturbation methods are applicable $[1,2,3,5]$. In this work we do not assume that the support is small. A priori estimates of solutions are proved in Section 3.

\section{Operators, spaces, topological degree}

\subsection{Operators and spaces}

We recall that the Leray-Schauder method, which is used here to prove the existence of waves, requires that the topological degree for the corresponding operators is defined. If this is the case, a homotopy of a given operator to some model operator with known properties should be constructed, and a priori estimates of solutions in the process of this deformation of the operator should be obtained.

When we consider unbounded domains, we should use some specially chosen weighted spaces. Otherwise, the degree with the usual properties may not exist. In this work we use weighted Hölder spaces $C_{\mu}^{k+\alpha}(\mathbb{R})$ with the norm

$$
\|u\|_{C_{\mu}^{k+\alpha}(\mathbb{R})}=\|u \mu\|_{C^{k+\alpha}(\mathbb{R})},
$$

where $k$ is a non-negative integer, $0<\alpha<1, C^{k+\alpha}(\mathbb{R})$ is the usual Hölder space. Parameter $\alpha$ here is different from the constant $\alpha$ introduced in (1.1). The weight function $\mu(x)$ has a polynomial growth at infinity. We take $\mu(x)=1+x^{2}$.

Obviously, any function $u \in C_{\mu}^{k+\alpha}(\mathbb{R})$ tends to zero at infinity. On the other hand, we look for solutions of equation (1.3) with the limits (1.4). Therefore, we introduce an infinitely differentiable function $\psi(x)$ such that $\psi(x)=w_{+}$for $x \geqslant 1$ and $\psi(x)=w_{-}$for $x \leqslant-1$ and put $w=u+\psi$. Hence we can write equation (1.3) in the form

$$
(u+\psi)^{\prime \prime}+c(u+\psi)^{\prime}+J(u+\psi)(u+\psi)(1-u-\psi)-\alpha(u+\psi)=0 .
$$

Consider the operator $A(u)$ corresponding to the left-hand side of the previous equation,

$$
A(u)=(u+\psi)^{\prime \prime}+c(u+\psi)^{\prime}+J(u+\psi)(u+\psi)(1-u-\psi)-\alpha(u+\psi)
$$


and acting in weighted Hölder spaces, $A: C_{\mu}^{2+\alpha}(\mathbb{R}) \rightarrow C_{\mu}^{\alpha}(\mathbb{R})$.

\subsection{Homotopy}

In [2] the authors have proved the existence of solutions in the form of monotone travelling waves for a class of integro-differential equations where the function $\phi$ has a small support $\left[-\varepsilon_{0}, \varepsilon_{0}\right]$. This result uses the bistability of the nonlinearity. It is applicable for the equation studied in this work. In this section we construct a homotopy that relates equation (1.3) to the equation with function $\phi$ having a small support. Let us introduce a family of operators $A_{\tau}: C_{\mu}^{2+\alpha}(\mathbb{R}) \times[0,1] \rightarrow C_{\mu}^{\alpha}(\mathbb{R})$ :

$$
A_{\tau}(u)=(u+\psi)^{\prime \prime}+c(u+\psi)^{\prime}+J_{\tau}(u+\psi)(u+\psi)(1-u-\psi)-\alpha(u+\psi)
$$

with

$$
J_{\tau}(u)=\int_{-\infty}^{\infty} \phi_{\tau}(x-y) u(y) d y
$$

where

$$
\phi_{\tau}(x)=\frac{\left(\varepsilon_{0}-1\right) \tau+1}{\varepsilon_{0}} \phi\left(\frac{\left(\left(\varepsilon_{0}-1\right) \tau+1\right) x}{\varepsilon_{0}}\right) .
$$

We study the following equation,

$$
w^{\prime \prime}+c w^{\prime}+J_{\tau}(w) w(1-w)-\alpha w=0
$$

If $\tau=0$ then we obtain operator $A_{0}$ with function $\phi(x)$, which has a small support, and, thus, the existence of a solution of equation $A_{0}(u)=0$ is known [3]. When $\tau=1$ we obtain equation (1.3).

Linearization of the operator $A_{\tau}$, introduced in (2.3), about the function $u_{1}(x)$ gives

$$
L_{\tau} u=\lim _{t \rightarrow 0} \frac{A_{\tau}\left(u_{1}+t u\right)-A_{\tau} u_{1}}{t}=u^{\prime \prime}+c u^{\prime}+J_{\tau}\left(w_{1}\right)\left(1-2 w_{1}\right) u+w_{1}\left(1-w_{1}\right) J_{\tau}(u)-\alpha u,
$$

where $w_{1}=u_{1}+\psi$. Let us introduce limiting operators of operator $L$,

$$
L_{\tau \pm} u=u^{\prime \prime}+c u^{\prime}+w_{ \pm}\left(1-2 w_{ \pm}\right) u+w_{ \pm}\left(1-w_{ \pm}\right) J_{\tau}(u)-\alpha u .
$$

Let us recall the definition of a proper operator and of Condition NS.

Definition 1 (Properness). Operator $A(u): E_{0} \rightarrow E$ is proper if the intersection of the inverse image of a compact set with any bounded closed ball $B \in E_{0}$ is compact.

Definition 2 (Condition NS). We say that the operator L satisfies Condition NS if the limiting equations $L_{ \pm} u=0$ do not have nonzero solutions in $C^{2+\alpha}(\mathbb{R})$.

Lemma 1 (Schauder estimate). For any solution u of equation $L_{\tau} u=f \in C^{\alpha}(\mathbb{R})$, the following estimate holds:

$$
\|u\|_{C^{2+\alpha}(\mathbb{R})} \leqslant K\left(\left\|L_{\tau} u\right\|_{C^{\alpha}(\mathbb{R})}+\|u\|_{C(\mathbb{R})}\right)
$$

where $K$ is a constant.

Proof. The proof of this lemma for a similar integro-differential operator can be found in [3]. 


\subsection{Fredholm operator with zero index}

We recall that an operator satisfies the Fredholm property if it is normally solvable, its kernel has a finite dimension and the codimension of its image is also finite. Elliptic operators in unbounded domains are normally solvable with a finite dimensional kernel if the Condition NS is satisfied [16]. Invertibility of limiting operators provides the Fredholm property.

The operator $L_{\tau}$ acting from $C^{2+\alpha}(\mathbb{R})$ into $C^{\alpha}(\mathbb{R})$ satisfies the Fredholm property and has zero index. The proof of this assertion follows the same lines as for elliptic operators (cf. [3]). We should verify that the Fredholm property remains valid in the weighted spaces, $L_{\tau}: C_{\mu}^{2+\alpha}(\mathbb{R}) \rightarrow$ $C_{\mu}^{\alpha}(\mathbb{R})$, and the index equals zero. From Lemma 2.24 in [15] it follows that it is sufficient to verify that the operator

$$
K u=\mu L_{\tau} u-L_{\tau}(\mu u), \quad K: C_{\mu}^{2+\alpha}(\mathbb{R}) \rightarrow C^{\alpha}(\mathbb{R})
$$

is compact. Consider a sequence $\left\{u_{i}\right\}$ such that $\left\|u_{i}\right\|_{C_{\mu}^{2+\alpha}(\mathbb{R})} \leqslant M$. We should prove that from the sequence $\left\{K u_{i}\right\}$ we can find a convergent in $C^{\alpha}(\mathbb{R})$ subsequence. Put $v_{i}=u_{i} \mu$. Then $\left\|v_{i}\right\|_{C^{2+\alpha}(\mathbb{R})} \leqslant M$. From the sequence $\left\{v_{i}\right\}$ we can find a subsequence, denoted again by $\left\{v_{i}\right\}$, convergent locally in $C^{2}$ to some function $v_{0}$. Denote $z_{i}=v_{i}-v_{0}$, then

$$
\left\|K u_{i}-K u_{0}\right\|_{C^{\alpha}(\mathbb{R})}=\left\|K \frac{z_{i}}{\mu}\right\|_{C^{\alpha}(\mathbb{R})}
$$

where $\left\|z_{i}\right\|_{C^{2+\alpha}(\mathbb{R})} \leqslant M_{1}, z_{i} \rightarrow 0$ in $C^{2}$ locally. Denote $y_{i}=K\left(z_{i} / \mu\right)$. We should prove that $\left\|y_{i}\right\|_{C^{\alpha}} \rightarrow 0$. The definition of the operator $K$ gives

$$
\begin{array}{r}
y_{i}=\mu L_{\tau}\left(z_{i} / \mu\right)-L_{\tau} z_{i}= \\
\left(-z_{i} \frac{\mu^{\prime \prime}}{\mu}-2 z_{i}^{\prime} \frac{\mu^{\prime}}{\mu}+2 z_{i}\left(\frac{\mu^{\prime}}{\mu}\right)^{2}-c z_{i} \frac{\mu^{\prime}}{\mu}\right)+w_{1}\left(1-w_{1}\right)\left(\mu J_{\tau}\left(z_{i} / \mu\right)-J_{\tau}\left(z_{i}\right)\right) .
\end{array}
$$

We have

$$
\mu J_{\tau}\left(z_{i} / \mu\right)-J_{\tau}\left(z_{i}\right)=\int_{-\infty}^{\infty} \phi(\xi) z_{i}(x-\xi)\left(\frac{\mu(x)}{\mu(x-\xi)}-1\right) d \xi .
$$

Both terms in the right-hand side of (2.10) uniformly tend to zero at infinity. From this and from the local convergence $z_{i} \rightarrow 0$ in $C^{2}$, it follows that $y_{i}$ converges to zero in $C^{\alpha}(\mathbb{R})$.

\subsection{Properness}

In this section we show that the operator $A_{\tau}$, defined in (2.3), is proper. First, let us prove the following lemma.

Lemma 2. For any $u, u_{0} \in C_{\mu}^{2+\alpha}$ and $\tau, \tau_{0} \in[0,1]$ the following representation holds

$$
A_{\tau}(u)-A_{\tau_{0}}\left(u_{0}\right)=A_{\tau_{0}}^{\prime}\left(u_{0}\right)\left(u-u_{0}\right)+\varphi\left(u, u_{0}, \tau, \tau_{0}\right),
$$


where $A_{\tau_{0}}^{\prime}\left(u_{0}\right)\left(u-u_{0}\right)=L_{\tau_{0}}\left(u_{0}\right)\left(u-u_{0}\right)$ and

$$
\varphi\left(u, u_{0}, \tau, \tau_{0}\right)=J_{\tau}(w) w(1-w)-J_{\tau_{0}}(w) w_{0}\left(1-w_{0}\right)-J_{\tau_{0}}\left(w_{0}\right)\left(1-2 w_{0}\right)\left(w-w_{0}\right),
$$

where $w=u+\psi$ and $w_{0}=u_{0}+\psi$. Moreover

$$
\varphi\left(u, u_{0}, \tau_{0}, \tau_{0}\right)=\left(u-u_{0}\right)\left(J_{\tau_{0}}(w)\left(1-w-w_{0}\right)-J_{\tau_{0}}\left(w_{0}\right)\left(1-2 w_{0}\right)\right) .
$$

Proof. Let us denote the difference $A_{\tau}(u)-A_{\tau_{0}}\left(u_{0}\right)-L_{\tau_{0}}\left(u_{0}\right)\left(u-u_{0}\right)$ by $\varphi\left(u, u_{0}, \tau, \tau_{0}\right)$, then using (2.3) and (2.7) we obtain

$$
\begin{gathered}
\varphi\left(u, u_{0}, \tau, \tau_{0}\right)=\left(u-u_{0}\right)^{\prime \prime}+c\left(u-u_{0}\right)^{\prime}+J_{\tau}(w) w(1-w)-J_{\tau_{0}}\left(w_{0}\right) w_{0}\left(1-w_{0}\right)-\alpha\left(u-u_{0}\right)- \\
\left(u-u_{0}\right)^{\prime \prime}-c\left(u-u_{0}\right)^{\prime}-J_{\tau_{0}}\left(w_{0}\right)\left(1-2 w_{0}\right)\left(w-w_{0}\right)-w_{0}\left(1-w_{0}\right) J_{\tau_{0}}\left(w-w_{0}\right)+\alpha\left(u-u_{0}\right)= \\
J_{\tau}(w) w(1-w)-J_{\tau_{0}}\left(w_{0}\right) w_{0}\left(1-w_{0}\right)-J_{\tau_{0}}\left(w_{0}\right)\left(1-2 w_{0}\right)\left(w-w_{0}\right)-w_{0}\left(1-w_{0}\right) J_{\tau_{0}}\left(w-w_{0}\right) \\
=J_{\tau}(w) w(1-w)-J_{\tau_{0}}(w) w_{0}\left(1-w_{0}\right)-J_{\tau_{0}}\left(w_{0}\right)\left(1-2 w_{0}\right)\left(w-w_{0}\right) .
\end{gathered}
$$

Simple transformation of $\varphi\left(u, u_{0}, \tau_{0}, \tau_{0}\right)$ gives (2.12). The lemma is proved.

Theorem 2 (properness of operator $A_{\tau}$ ). If the operator $L_{\tau}$, defined in (2.7), satisfies Condition $N S$, then the operator $A_{\tau}(u)$, defined in (2.3), is proper with respect to both $u$ and $\tau$.

Proof. Consider a convergent in $C_{\mu}^{\alpha}(\mathbb{R})$ sequence $\left\{f_{n}\right\} \rightarrow f_{0}$ and solutions of the equations

$$
A_{\tau_{n}}\left(u_{n}\right)=f_{n}
$$

bounded in $C_{\mu}^{2+\alpha}(\mathbb{R}) \times[0,1],\left\|u_{n}\right\|_{C_{\mu}^{2+\alpha}(\mathbb{R})} \leqslant M$. We should prove the existence of a subsequence $\left\{u_{m_{n}}\right\}$ that is convergent in $C_{\mu}^{2+\alpha}(\mathbb{R})$ to a function $u_{0} \in C_{\mu}^{2+\alpha}(\mathbb{R})$.

Since $\left\{u_{n}\right\}$ is bounded in $C_{\mu}^{2+\alpha}(\mathbb{R})$, it admits a subsequence $\left\{u_{n_{m}}\right\}$, which is convergent to a function $u_{0}$, uniformly on any bounded interval $I \subset \mathbb{R}$. By a diagonalisation process we prolong $u_{0}$ to all $\mathbb{R}$. The limit function $u_{0} \in C_{\mu}^{2+\alpha}(\mathbb{R})$. Passing to the limit $m \rightarrow \infty$ in the equation $A_{\tau_{n_{m}}}\left(u_{n_{m}}\right)=f_{n_{m}}$ we obtain

$$
A_{\tau_{0}}\left(u_{0}\right)=f_{0} .
$$

We know that $u_{n_{m}} \rightarrow u_{0}$ uniformly on any bounded interval $I$. Let us show that the convergence is uniform with respect to $x$ on the whole axis, $u_{n_{m}} \rightarrow u_{0}$ in $C(\mathbb{R})$.

Let us write the subscript $n$ instead of $n_{m}$ and denote

$$
v_{n}=u_{n} \mu, \quad v_{0}=u_{0} \mu, \quad z_{n}=v_{n}-v_{0}, \quad g_{n}=f_{n} \mu, \quad g_{0}=f_{0} \mu .
$$

Subtracting (2.14) from (2.13) and multiplying it by $\mu$ we obtain

$$
\left(A_{\tau_{n}}\left(u_{n}\right)-A_{\tau_{0}}\left(u_{n}\right)\right) \mu+\left(A_{\tau_{0}}\left(u_{n}\right)-A_{\tau_{0}}\left(u_{0}\right)\right) \mu=g_{n}-g_{0} .
$$

Denote

$$
r_{n}=\left(A_{\tau_{n}}\left(u_{n}\right)-A_{\tau_{0}}\left(u_{n}\right)\right) \mu,
$$


then we have

$$
\left\|r_{n}\right\|_{C^{\alpha}(\mathbb{R})} \rightarrow 0, \quad n \rightarrow \infty
$$

Injecting (2.15), (2.17), and (2.3) into (2.16) and taking into account Lemma 2, we obtain

$$
\begin{aligned}
& z_{n}^{\prime \prime}+z_{n}^{\prime}+J_{\tau_{0}}\left(w_{0}\right)\left(1-2 w_{0}\right) z_{n}+w_{0}\left(1-w_{0}\right) J_{\tau_{0}}\left(u_{n}-u_{0}\right) \mu-\alpha z_{n}+ \\
& \quad\left(-z_{n} \frac{\mu^{\prime \prime}}{\mu}-2 z_{n}^{\prime} \frac{\mu^{\prime}}{\mu}+2 z_{n}\left(\frac{\mu^{\prime}}{\mu}\right)^{2}-c z_{n} \frac{\mu^{\prime}}{\mu}\right)+\varphi\left(u_{n}, u_{0}, \tau_{0}, \tau_{0}\right) \mu=g_{n}-g_{0}-r_{n} .
\end{aligned}
$$

We should prove that there exists a subsequence $\left\{z_{n_{k}}\right\}$ convergent uniformly on the whole axis, $z_{n_{k}} \rightarrow 0$ in $C(\mathbb{R})$. Suppose that it is not true. Then there exists an unbounded sequence $\left\{x_{m}\right\}$, such that $\left|z_{m}\left(x_{m}\right)\right| \geqslant \varepsilon>0$. Let us introduce shifted functions,

$$
\tilde{z}_{m}(x)=z_{m}\left(x+x_{m}\right) .
$$

Since $\left\|\tilde{z}_{m}\right\|_{C^{2+\alpha}(\mathbb{R})} \leqslant M$, from sequence $\left\{\tilde{z}_{m}\right\}$ we can choose a subsequence $\left\{\tilde{z}_{m_{k}}(x)\right\}$ converging to a limiting function $\tilde{z}_{0} \in C^{2+\alpha}(\mathbb{R})$ in $C^{2}$ uniformly on any bounded interval $I \subset \mathbb{R}$ (the same reasoning as above). We have $\left|\tilde{z}_{0}(0)\right| \geqslant \varepsilon>0$. Functions $\tilde{z}_{m_{k}}(x)$ with shifted arguments satisfy equation (2.18). From the definition of the weight function $\mu$ we obtain

$$
\frac{\mu^{\prime}\left(x+x_{m}\right)}{\mu\left(x+x_{m}\right)} \rightarrow 0, \quad \frac{\mu^{\prime \prime}\left(x+x_{m}\right)}{\mu\left(x+x_{m}\right)} \rightarrow 0 \quad \text { as } \quad x_{m} \rightarrow \infty .
$$

From (2.12):

$$
\varphi\left(u_{m}\left(x+x_{m}\right), u_{0}\left(x+x_{m}\right), \tau_{0}, \tau_{0}\right) \mu=z_{m}(x) T_{m}\left(x+x_{m}\right),
$$

where

$$
\begin{gathered}
T_{m}(y)=J_{\tau_{0}}\left(w_{m}(y)\right)\left(1-w_{m}(y)-w_{0}(y)\right)-J_{\tau_{0}}\left(w_{0}(y)\right)\left(1-2 w_{0}(y)\right)= \\
\left(J_{\tau_{0}}\left(w_{m}(y)-w_{0}(y)\right)\right)\left(1-w_{m}(y)-w_{0}(y)\right)+J_{\tau_{0}}\left(w_{0}(y)\right)\left(w_{0}(y)-w_{m}(y)\right)= \\
\left(J_{\tau_{0}}\left(z_{m} / \mu\right)\left(1-w_{m}(y)-w_{0}(y)\right)-J_{\tau_{0}}\left(w_{0}(y)\right) z_{m} / \mu .\right.
\end{gathered}
$$

Here $w_{m}=u_{m}+\psi$ Since $z_{m} \rightarrow 0$ in $C^{2}$ uniformly in every bounded interval, then $z_{m} / \mu \rightarrow 0$ in $C^{2}(\mathbb{R})$. Hence

$$
\left\|\varphi\left(u_{m}\left(x+x_{m}\right), u_{0}\left(x+x_{m}\right), \tau_{0}, \tau_{0}\right) \mu\right\|_{C^{\alpha}} \rightarrow 0 \quad \text { as } \quad m \rightarrow \infty .
$$

By definition of $g_{m}$ and $g_{0},\left\|g_{m}-g_{0}\right\|_{C^{\alpha}(\mathbb{R})} \rightarrow 0$ as $m \rightarrow \infty$. In the limit $m \rightarrow \infty$, equation (2.18) becomes

$$
\widehat{L} \tilde{z}_{0}=0 \text {, }
$$

which means that limiting operator $\widehat{L}$ admits a nonzero solution. This contradicts Condition NS. Thus, convergence $z_{m} \rightarrow 0$ is uniform in $C(\mathbb{R})$. Using this convergence and the Schauder estimate (Lemma 1) we obtain convergence $z_{m} \rightarrow 0$ in $C^{2+\alpha}(\mathbb{R})$, which means the convergence $u_{m} \rightarrow u_{0}$ in $C_{\mu}^{2+\alpha}(\mathbb{R})$. This completes the proof. 


\subsection{Functionalisation of parameter $c$}

We recall that parameter $c$ in (1.3) is an unknown constant that should be found along with function $w(x)$. Note that solutions of (1.3) are invariant with respect to translation, i.e. if $w(x)=u(x)+$ $\psi(x)$ is a solution of (1.3), then $w(x+h)=u(x+h)+\psi(x+h)$ is also a solution of this equation for all $h \in \mathbb{R}$. Then the weighted norm $\|u(x+h) \mu(x)\|_{C^{2+\alpha}}$ tends to infinity as $h \rightarrow \pm \infty$. Hence the set of solutions of the equation $A(u)=0$ is not bounded in the space $C_{\mu}^{2+\alpha}(\mathbb{R})$, and the topological degree cannot be applied.

To get rid of the invariance of solutions with respect to translation, we apply functionalisation of parameter $c$ first used for travelling waves in $[12,13]$. We introduce a functional $c=c(u)$ that satisfies the following properties:

1. $c(u)$ satisfies Lipschitz condition on every bounded set in $C_{\mu}^{2+\alpha}(\mathbb{R})$ and has a continuous Fréchet derivative,

2. function $\tilde{c}(h)=c(u(x+h))$ is a decreasing function of $h$, such that $\tilde{c}(-\infty)=+\infty$ and $\tilde{c}(+\infty)=-\infty$,

3. the solution $w=u+\psi$ of equation (1.3) satisfies

$$
\left\langle c^{\prime}(u), w^{\prime}\right\rangle \neq 0 .
$$

We introduce the following function

$$
c(u)=\ln \int_{R}\left(u(x)+\psi(x)-w_{+}\right)^{2} \sigma(x) d x,
$$

where $\sigma(x)$ is an increasing function, with $\sigma(-\infty)=0, \sigma(+\infty)=1$ and

$$
\int_{-\infty}^{0} \sigma(x) d x<\infty .
$$

The function $c(u)$ introduced in this way satisfies the conditions enumerated above, the proof can be found in [13]. Equation (1.3) is equivalent to the equation

$$
(u+\psi)^{\prime \prime}+c(u)(u+\psi)^{\prime}+J(u+\psi)(u+\psi)(1-u-\psi)-\alpha(u+\psi)=0 .
$$

Instead of the operator $A_{\tau}$ introduced in Section 2.2 we will consider the operator

$$
A_{\tau}(u)=(u+\psi)^{\prime \prime}+c(u)(u+\psi)^{\prime}+J_{\tau}(u+\psi)(u+\psi)(1-u-\psi)-\alpha(u+\psi),
$$

where the constant $c$ is replaced by the functional $c(u)$. We keep for it the same notation. It can be easily verified that introduction of the functional $c(u)$ does not change the Fredholm property and the properness of the operator. 


\subsection{Construction of topological degree}

Consider an operator $A$ acting from a Banach space $E$ into another Banach space $F$. By definition, topological degree is an integer number $\gamma(A, D)$ which depends on the operator and on the domain $D$ in the function space $E$. Topological degree for elliptic operators in unbounded domains is constructed in $[15,14]$ on the basis of the theory of Fredholm and proper operators. The results on the Fredholm property and properness of the integro-differential operators presented above allow us to use the same construction.

One of the main applications of the topological degree is related to the Leray-Schauder method widely used to prove existence of solutions of various problems. We briefly recall the main ideas of this method. If the operator $A_{\tau}$ continuously depends on the parameter $\tau$ and

$$
A_{\tau}(u) \neq 0, \quad u \in \partial D, \quad \tau \in[0,1],
$$

then the value $\gamma\left(A_{\tau}, D\right)$ of the degree does not depend on $\tau$. This property is called homotopy invariance. If we can reduce the operator $A=A_{1}$ by a continuous deformation to a model operator $A_{0}$ for which $\gamma\left(A_{0}, D\right) \neq 0$ and (2.20) is satisfied, then $\gamma(A, D) \neq 0$. Another property of the degree, nonzero rotation, ensures that the equation $A(u)=0$ has a solutions in the domain $D$.

Let us take as a domain $D$ a ball $B$ of the radius $R$. Then condition (2.20) will be satisfied if all solutions of the equation $A_{\tau}(u)=0$ satisfy the inequality $\|u\| \leqslant R$. These are a priori estimates of solutions. They play a crucial role in the proof of the existence of solutions. We discuss them in the next section.

\section{A priori estimates}

A priori estimates of travelling wave solutions have some specific features. We note first of all that they imply not only the estimate of the function $w(x)$ but also of the wave velocity. Moreover, the function $w(x)$ should be estimated in the weighted space. We consider in fact the function $u(x)=w(x)-\psi(x)$ with the zero limits at infinity. It decays exponentially at infinity, so its weighted norm with a polynomial weight is limited. The difficulty is to estimate it "far" from infinity.

To explain the origin of this difficulty, let us consider the following geometrical interpretation. Travelling wave is a heteroclinic trajectory of some first order ordinary differential system of equations. Suppose that this trajectory approaches some intermediate stationary point during the deformation of the system. In the limit we can obtain two heteroclinic trajectories which connect consecutively three stationary points. This situation corresponds to loss of a priori estimates in the weighted space. Thus, we need to prove that the trajectory, which corresponds to the travelling wave, cannot approach intermediate stationary points or other invariant manifolds.

We will follow here the method developed in $[12,13]$ for monotone reaction-diffusion systems. It consists of two steps. First of all, we separate monotone and non-monotone solutions $w(x)$ of problem (1.3), (1.4). This means that two sequences of solutions, $w_{M}^{j}(x)$ and $w_{N}^{j}(x)$, where the former are monotone with respect to $x$ and the latter non-monotone, cannot converge in $C^{2}(\mathbb{R})$ to the same limiting function. This result allows us to deal only with monotone solutions. At the 
second step, we obtain a priori estimates of monotone solutions. According to the geometrical interpretation given above, we prove that the trajectory cannot be attracted by an intermediate stationary point. This will follow from the sign of the wave velocity. Here we use the monotonicity of solutions. Otherwise, the intermediate manifold can have a more complex structure, and the method is not applicable.

\subsection{Monotonicity}

In this section we show that monotone solutions of equation (2.6) are strictly monotone. We begin with an auxiliary result on the positiveness of solutions of the linear parabolic equation

$$
\frac{\partial v}{\partial t}=\frac{\partial^{2} v}{\partial x^{2}}+a(x, t) J(v)+b(x, t) v
$$

in $x \in \mathbb{R}$ assuming that $a(x, t)$ and $b(x, t)$ are continuous functions, $a(x, t) \geqslant 0$. We cannot directly use the classical positiveness theorems here because of the integral term in the right-hand side.

Lemma 3. Suppose that $a(x, t)+b(x, t)<0$ for $|x| \geqslant N, 0 \leqslant t \leqslant T$ with some positive $N$ and T. If $v(x, 0) \geqslant 0$ and $v(x, 0) \not \equiv 0$, then $v(x, t)>0$ for $0 \leqslant t \leqslant T$.

Proof. Suppose that the assertion of the lemma does not hold. If there exists such $\left(x_{0}, t_{0}\right)$ that $v\left(x_{0}, t_{0}\right)=0, v\left(x, t_{0}\right) \geqslant 0$ for all $x \in \mathbb{R}$, and $v(x, t)>0$ for all $x \in \mathbb{R}$ and $0<t<t_{0}$, then we obtain a contradiction with the classical positiveness theorem [7]. Indeed, we can write equation (3.1) in the form

$$
\frac{\partial v}{\partial t}=\frac{\partial^{2} v}{\partial x^{2}}+b(x, t) v+c(x, t)
$$

where $c(x, t)=a(x, t) J(v) \geqslant 0$ for all $x$ and $0 \leqslant t \leqslant t_{0}$. Since $v(x, 0)$ is non-negative and not identically zero, then $v\left(x, t_{0}\right)$ should be strictly positive.

Otherwise, the solution becomes negative for some $t_{1}>0$. Then there exists such $t_{0}, 0 \leqslant t_{0} \leqslant$ $t_{1}$ that $v\left(x, t_{0}\right) \geqslant 0$ for all $x \in \mathbb{R}$ and $v\left(x_{k}, t_{k}\right)<0$ for some sequences $x_{k}$ and $t_{k}>t_{0}, t_{k} \rightarrow t_{0}$ as $k \rightarrow \infty$. If the sequence $x_{k}$ is bounded, then we can find a convergent subsequence. Denote its limit by $x_{0}$. Then $v\left(x, t_{0}\right) \geqslant 0$ for all $x$ and $v\left(x_{0}, t_{0}\right)=0$. As above, we obtain the contradiction with the classical positiveness theorem.

Hence, the sequence $x_{k}$ does not have bounded limiting points. Without loss of generality we can assume that $x_{k} \rightarrow+\infty$. For each fixed $t$ we have $v(x, t) \rightarrow 0$ as $x \rightarrow \infty$. Therefore, the function $v\left(x, t_{k}\right)$ has a negative minimum that we denote by $x_{k}^{m}$. As before, $x_{k}^{m} \rightarrow+\infty$.

Let us choose $k$ sufficiently large such that $x_{k}^{m}>N$. Put $z=v+\epsilon$ where $\epsilon=\left|v\left(x_{k}^{m}, t_{k}\right)\right|$. Then $z$ satisfies the equation

$$
\frac{\partial z}{\partial t}=\frac{\partial^{2} z}{\partial x^{2}}+a(x, t) J(z)+b(x, t) z-(a(x, t)+b(x, t)) \epsilon .
$$

Moreover, $z\left(x, t_{k}\right) \geqslant 0$ for all $x, 0 \leqslant t \leqslant t_{k}$ and $z\left(x_{k}, t_{k}\right)=0$. We obtain a contradiction in signs in the last equation. Indeed, the time derivative at the point $x=x_{k}, t=t_{k}$ is non-positive, while 
all terms in the right-hand side are non-negative. The last term is strictly positive. The lemma is proved.

Lemma 4. If $w_{0}(x)$ is a non-constant solution of equation (2.6) such that $w_{0}^{\prime}(x) \leqslant 0$ for all $x \in \mathbb{R}$, then $w_{0}^{\prime}(x)<0$.

Proof. Denote $v=-w^{\prime}$. Differentiating equation (2.6) and taking into account that $J^{\prime}(w)=J(v)$, we obtain

$$
v^{\prime \prime}+c v^{\prime}+a(x) J(v)+b(x) v=0,
$$

where

$$
a(x)=w(1-w), \quad b(x)=J(w)(1-2 w)-\alpha .
$$

We note that $a(x)+b(x)<0$ for $|x|$ sufficiently large. Indeed, $a(x)+b(x) \rightarrow F^{\prime}\left(w_{ \pm}\right)$as $x \rightarrow \pm \infty$. According to the conditions of Section $1, F^{\prime}\left(w_{ \pm}\right)<0$.

We should prove that the solution of this equation, which satisfies $v \geqslant 0$, is strictly positive. This follows from Lemma 3 if we take function $v(x)$ as initial condition.

Lemma 5. If $w_{j}(x)$ is a sequence of solutions of problem (2.6), (1.4) such that $w_{j} \rightarrow w_{0}$ in $C^{1}(\mathbb{R})$, where $w_{0}^{\prime}(x) \leqslant 0, x \in \mathbb{R}$, then for all $j$ sufficiently large $0<w_{j}(x)<1$ and $w_{j}^{\prime}(x)<0, x \in \mathbb{R}$.

Proof. Let us first prove the inequality $0<w_{j}(x)<1$. The right inequality holds for $j$ sufficiently large because of the uniform convergence $w_{j} \rightarrow w_{0}$ and $0<w_{0}(x)<w_{-}<1$. We now verify that $w_{j}(x)$ is positive for all $x$. If this is not the case, then each of these functions has a negative minimum $x_{j}$. From the uniform convergence $w_{j} \rightarrow w_{0}$ it follows that $x_{j} \rightarrow+\infty$ and $J\left(w_{j}\left(x_{j}\right)\right) \rightarrow$ 0 . Hence,

$$
w_{j}^{\prime \prime}\left(x_{j}\right) \geqslant 0, \quad w_{j}^{\prime}\left(x_{j}\right)=0, \quad J\left(w_{j}\left(x_{j}\right)\right)\left(1-w_{j}\left(x_{j}\right)\right)-\alpha<0, \quad w_{j}\left(x_{j}\right)<0 .
$$

This gives a contradiction in signs in the equation

$$
w_{j}^{\prime \prime}+c w_{j}^{\prime}+\left(J\left(w_{j}\right)\left(1-w_{j}\right)-\alpha\right) w_{j}=0 .
$$

Next, we prove that the functions $w_{j}$ are decreasing. Suppose that this assertion does not hold and there exists a sequence $x_{j}$ such that $w_{j}^{\prime}\left(x_{j}\right)=0$. If it is bounded, then there exists a subsequence converging to some point $x_{0}$. Hence $w_{0}^{\prime}\left(x_{0}\right)=0$. We obtain a contradiction with Lemma 4.

Consider now the case where $x_{j} \rightarrow+\infty$. Denote $v=-w^{\prime}$. Differentiating equation (2.6) and taking into account that $J^{\prime}(w)=J(v)$, we obtain

$$
v^{\prime \prime}+c v^{\prime}+a(x) J(v)+b(x) v=0,
$$

where

$$
a(x)=w(1-w), \quad b(x)=J(w)(1-2 w)-\alpha .
$$


If $v_{j}(x) \geqslant 0$ for all $x$, then the existence of $x_{j}$ such that $v_{j}\left(x_{j}\right)=0$ contradicts Lemma 4 . Therefore, there exist negative values of this function for each $j$. On the other hand, from the convergence $w_{j}^{\prime} \rightarrow w_{0}^{\prime}$ it follows that $v_{j}$ is positive in each given interval for $j$ sufficiently large. Finally, note that $v_{j}(x) \rightarrow 0$ as $x \rightarrow \pm \infty$. Hence, there exists a sequence $\hat{x}_{j} \rightarrow \infty$ such that it is a negative minimum of the function $v_{j}(x)$. The uniform convergence $w_{j}^{\prime}(x) \rightarrow w_{0}^{\prime}(x)$ implies that $v_{j}\left(\hat{x}_{j}\right) \rightarrow 0$ as $j \rightarrow \infty$.

Put $\epsilon=\left|v_{j}\left(\hat{x}_{j}\right)\right|$ for some $j$ sufficiently large and $z(x)=v_{j}(x)+\epsilon$. Then

$$
z^{\prime \prime}+c z^{\prime}+a(x) J(z)+b(x) z-(a(x)+b(x)) \epsilon=0 .
$$

Moreover, $z(x) \geqslant 0$ for $-\infty<x<\infty, z\left(x_{j}\right)=0$. Hence $z^{\prime \prime}\left(x_{j}\right) \geqslant 0, z^{\prime}\left(x_{j}\right)=0, J(z)\left(x_{j}\right) \geqslant 0$. Since $0<w_{j}(x)<1$, then $a\left(x_{j}\right)>0$. It remains to note that $a\left(x_{j}\right)+b\left(x_{j}\right)<0$ for $j$ sufficiently large. Indeed, if $x_{j} \rightarrow+\infty$, then $a\left(x_{j}\right) \rightarrow 0, b\left(x_{j}\right) \rightarrow-\alpha$. If $x_{j} \rightarrow-\infty$, then $a\left(x_{j}\right) \rightarrow \alpha$, $b\left(x_{j}\right) \rightarrow w_{-}\left(1-2 w_{-}\right)-\alpha$, that is

$$
a\left(x_{j}\right)+b\left(x_{j}\right) \rightarrow w_{-}\left(1-2 w_{-}\right)<0 .
$$

We obtain a contradiction in signs in equation (3.3). The lemma is proved.

\subsection{Estimates of derivatives}

In this section we obtain estimates in $C(-\infty,+\infty)$ of the derivatives $w^{\prime}(x)$ and $w^{\prime \prime}(x)$ of the solution $w(x)$ of equation (2.6). We will use the notation $R_{2}=\max _{|w| \leqslant R}\left|J_{\tau}(w) w(1-w)-\alpha w\right|$.

Lemma 6. Let solution $w(x)$ of equation (2.6) satisfy inequality $|w(x)| \leqslant R$ for all $x$. Then the derivatives $w^{\prime}(x)$ and $w^{\prime \prime}(x)$ can be estimated in $C(-\infty,+\infty)$ by a constant, depending only on $R$ and $R_{2}$.

Proof. Let us first obtain an estimate for the first derivative $w^{\prime}(x)$. We consider two cases, $|c| \geqslant 1$ and $|c|<1$. Suppose that $|c| \geqslant 1$. The second derivative $w^{\prime \prime}(x)$ equals zero when $w^{\prime}(x)$ is at its extremum point, which we denote by $x_{0}$. Then from equation (2.6) we obtain

$$
\left.\left|w^{\prime}(x)\right| \leqslant\left|w^{\prime}\left(x_{0}\right)\right| \leqslant \mid J_{\tau}\left(w\left(x_{0}\right)\right) w\left(x_{0}\right)\left(1-w\left(x_{0}\right)\right)-\alpha w\left(x_{0}\right)\right) \mid \leqslant R_{2}, \quad \forall x \in \mathbb{R} .
$$

Suppose now that $|c|<1$. Consider an interval $[\alpha, \beta]$ where $\left|w^{\prime}(x)\right| \geqslant 1$. Integrating equation (2.6) over the interval $[\alpha, x]$, we obtain

$$
w^{\prime}(x)-w^{\prime}(\alpha)+c(w(x)-w(\alpha))+\int_{\alpha}^{x}\left(J_{\tau}(w(x)) w(x)(1-w(x))-\alpha w(x)\right) d x=0 .
$$

This provides

$$
\left|w^{\prime}(x)\right| \leqslant 1+|c||w(x)-w(\alpha)|+R_{2}(\beta-\alpha) \leqslant 1+2 R+R_{2}(\beta-\alpha) .
$$


The mean-value theorem gives

$$
|w(\beta)-w(\alpha)|=\left|w^{\prime}\left(x_{0}\right)\right|(\beta-\alpha) \geqslant(\beta-\alpha),
$$

which provides that $\beta-\alpha \leqslant 2 R$ and thus finally we obtain

$$
\left|w^{\prime}(x)\right| \leqslant 1+2 R+2 R R_{2} .
$$

So we have estimated the first derivative of the function $w(x)$. Let us now estimate the second derivative. From equation (2.6) we obtain

$$
\left|w^{\prime \prime}(x)\right| \leqslant\left|c w^{\prime}(x)\right|+R_{2} .
$$

The first term in the right-hand side has its maximum when $w^{\prime}(x)$ reaches its extremum, that is $w^{\prime \prime}(x)=0$ at this point. Therefore, from equation (2.6)

$$
\left|c w^{\prime}(x)\right| \leqslant R_{2} \text {. }
$$

Hence

$$
\left|w^{\prime \prime}(x)\right| \leqslant 2 R_{2},
$$

which completes the proof of the lemma.

\subsection{A priori estimates of the speed}

In this section we obtain a priori estimates of the speed $c$ of monotone waves. Suppose that there exists a solution $w(x)$ of equation (1.3) for some $c=c_{0}$ with the limits (1.4) at infinity. Let us estimate the value $c_{0}$ of the speed. For this purpose we consider the evolution equation

$$
\frac{\partial u}{\partial t}=\frac{\partial^{2} u}{\partial x^{2}}+c_{0} \frac{\partial u}{\partial x}+J(u) u(1-u)-\alpha u .
$$

So that $w(x)$ is a stationary solution of this equation. Let $u(x, t)$ be a solution of equation (3.4) and $v=u-w_{0}$, where $w_{0}<1 / 2$ satisfies $w_{0}\left(1-w_{0}\right)=\alpha$. Then $v$ satisfies the equation

$$
\frac{\partial v}{\partial t}=\frac{\partial^{2} v}{\partial x^{2}}+c_{0} \frac{\partial v}{\partial x}+J(v)\left(1-w_{0}\right) u-J(u) u v .
$$

Suppose that $0<u<1$ and $v>0$. Then

$$
J(v)\left(1-w_{0}\right) u-J(u) u v<J(v) .
$$

Next, consider the equation

$$
\frac{\partial z}{\partial t}=\frac{\partial^{2} z}{\partial x^{2}}+c_{0} \frac{\partial z}{\partial x}+J_{\eta}(z),
$$




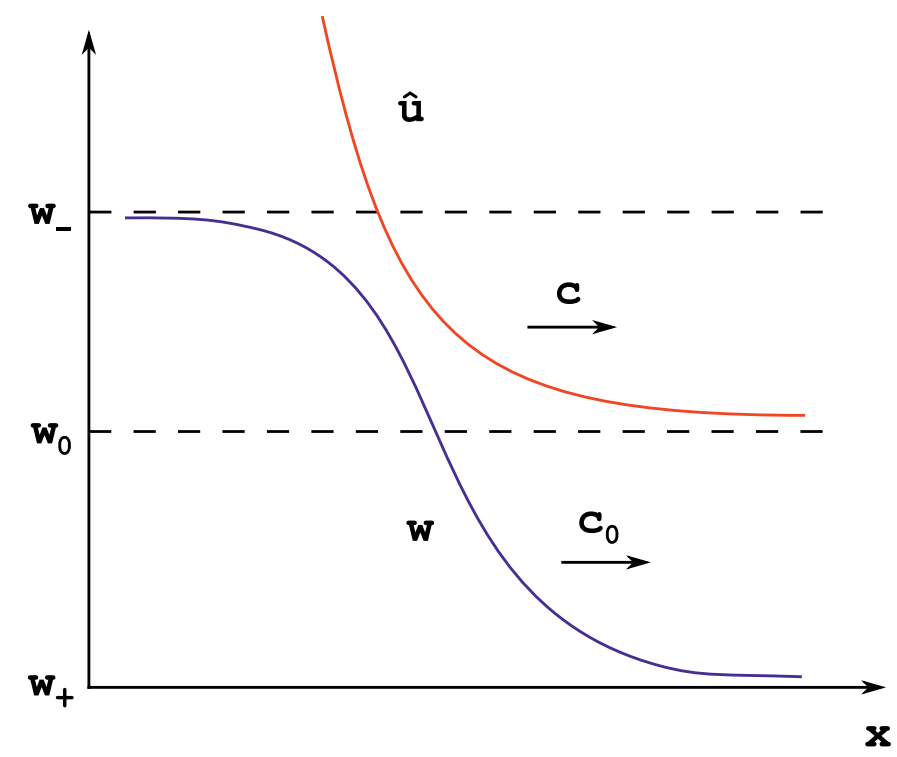

Figure 1: The estimate of the speed of the travelling wave solution of equation (1.1). The wave $w$ propagates with the speed $c_{0}$, the solution $\hat{u}$ with the speed $c$. The wave remains below the solution. If it touches it, this will contradict the comparison of solutions. Hence $c>c_{0}$.

where

$$
J_{\eta}(z)=\int_{-\infty}^{\infty} \eta(x-y) z(y, t) d y
$$

$\eta(x)$ is a piece-wise constant function equal to $M=\sup _{x} \phi(x)$ in the support of the function $\phi(x)$ and zero otherwise. Let the support of the function $\eta(x)$ be $[-N, N]$. Let us look for a solution of the equation

$$
w^{\prime \prime}+c w^{\prime}+J_{\eta}(w)=0
$$

for some $c$ possibly different from $c_{0}$ in the form of the exponential $w(x)=e^{-\lambda x}$. We obtain

$$
\lambda^{2}-c \lambda+\frac{M}{\lambda}\left(e^{\lambda N}-e^{-\lambda N}\right)=0 .
$$

For any $M$ and $N$, if $c$ is sufficiently large, then this equation has a solution $\lambda$. Let us take these values of $c$ and $\lambda$ and consider the corresponding solution $w(x)$ of equation (3.8). Then $z(x, t)=w\left(x-\left(c-c_{0}\right) t\right)$ is a solution of equation (3.7), which has a constant profile and moves to the right with the speed $\left(c-c_{0}\right)$. The function $\hat{u}(x, t)=z(x, t)+w_{0}$ satisfies the equation

$$
\frac{\partial \hat{u}}{\partial t}=\frac{\partial^{2} \hat{u}}{\partial x^{2}}+c_{0} \frac{\partial \hat{u}}{\partial x}+J_{\eta}\left(\hat{u}-w_{0}\right)
$$

Let us now compare the solution $u(x, t)$ of equation (3.4) and the solution $\hat{u}(x, t)$ of equation (3.9) (Figure 1). We recall that $u(x, t) \rightarrow w_{ \pm}$as $x \rightarrow \pm \infty$ for each $t \geqslant 0$ and $\hat{u}(x, t)$ is 
a strictly decreasing function converging to $w_{0}$ as $x \rightarrow+\infty$ and exponentially growing at $-\infty$. Hence, we can choose a constant $h$ such that $u(x, 0)<\hat{u}(x-h, 0)$ for all $x \in \mathbb{R}$. We are going to prove that $u(x, t)<\hat{u}(x-h, t)$ for all $x \in \mathbb{R}$ and $t \geqslant 0$. Suppose that this is not true. Then there exists $t_{0}>0$ such that $u\left(x, t_{0}\right) \leqslant \hat{u}\left(x-h, t_{0}\right)$ for all $x \in \mathbb{R}$ and $u\left(x_{0}, t_{0}\right)=\hat{u}\left(x_{0}-h, t_{0}\right)$ for some $x_{0}$. Since $\hat{u}\left(x_{0}-h, t_{0}\right)>w_{0}$, then $v\left(x_{0}, t_{0}\right)>0$. This inequality holds in some neighborhood $\delta\left(x_{0}\right)$ of $x=x_{0}$. Moreover, $0<u(x, t)<1, J(u)>0$. Hence, by virtue of (3.6),

$J(u) u(1-u)-\alpha u=J(v)\left(1-w_{0}\right) u-J(u) u v<J(v)=J\left(u-w_{0}\right) \leqslant J_{\eta}\left(\hat{u}-w_{0}\right), \quad x \in \delta\left(x_{0}\right)$.

We obtain a contradiction with the classical positiveness theorem. Thus, if there exists a stationary solution $w(x)$ of equation (3.4), then we put $u(x, 0)=w(x)$ and obtain $w(x) \equiv u(x, t)<\hat{u}(x, t)$. Therefore, $c_{0}<c$, where $c$ is the value of the speed chosen above.

In order to estimate the speed $c$ from below, we repeat a similar construction with a solution $w(x)$ such that $w(x) \rightarrow w_{0}$ as $x \rightarrow \infty$ and exponentially decreasing as $x \rightarrow+\infty$. It propagates to the left with a certain speed $\left(c-c_{0}\right)$. We thus have proved the following theorem.

Theorem 3. For any arbitrary solution $(c, w)$ of problem (2.6), (1.4), where $w(x)$ is monotonically decreasing function, there exists an estimate of the speed $c$ independent of $\tau$.

\subsection{Sign of the speed}

In this section we consider behaviour of solutions of equation (2.6) as $x \rightarrow \pm \infty$. The subscript $\tau$ is omitted. We prove that the waves connecting a stable point with an unstable point can exist only with the speed of a certain sign. This will be used below in order to obtain a priori estimates of solutions. We begin with an auxiliary result.

Lemma 7. Suppose that $v(x)$ is a decreasing positive function, $\phi(x)$ is even and non-negative. Then for any $N$

$$
\int_{N}^{\infty} d x \int_{-\infty}^{\infty} \phi(x-y) v(y) d y \geqslant \int_{N}^{\infty} d x \int_{-\infty}^{\infty} \phi(x-y) v(x) d y .
$$

If $v$ is a positive and increasing function, then

$$
\int_{-\infty}^{N} d x \int_{-\infty}^{\infty} \phi(x-y) v(y) d y \geqslant \int_{-\infty}^{N} d x \int_{-\infty}^{\infty} \phi(x-y) v(x) d y .
$$

It is assumed that all these integrals exist.

Proof. We have

$$
\int_{N}^{\infty} d x \int_{N}^{\infty} \phi(x-y) v(y) d y=\int_{N}^{\infty} d x \int_{N}^{\infty} \phi(x-y) v(x) d y .
$$

If $v$ is decreasing, then

$$
\int_{N}^{\infty} d x \int_{-\infty}^{N} \phi(x-y) v(y) d y \geqslant \int_{N}^{\infty} d x \int_{-\infty}^{N} \phi(x-y) v(x) d y
$$


since $y \leqslant N \leqslant x$ in the domain of integration and, consequently, $v(y) \geqslant v(N) \geqslant v(x)$. Taking a sum of the last two relations, we obtain (3.10).

Consider now the second case. From the equality

$$
\int_{-\infty}^{N} d x \int_{-\infty}^{N} \phi(x-y) v(y) d y=\int_{-\infty}^{N} d x \int_{-\infty}^{N} \phi(x-y) v(x) d y
$$

and inequality

$$
\int_{-\infty}^{N} d x \int_{N}^{\infty} \phi(x-y) v(y) d y \geqslant \int_{-\infty}^{N} d x \int_{N}^{\infty} \phi(x-y) v(x) d y,
$$

which takes place since in the domain of integration $x \leqslant N \leqslant y$ and $v(x) \leqslant v(N) \leqslant v(y)$, we obtain (3.11). The lemma is proved.

Let $w_{0}<1 / 2$ be a solution of the equation $u(1-u)=\alpha$. Suppose that there exists a decreasing solution $u(x)$ of equation (2.6) such that $u(x) \rightarrow w_{0}$ as $x \rightarrow+\infty$. Put $u(x)=w_{0}+v(x)$. Then

$$
v^{\prime \prime}+c v^{\prime}+\left(J(v)\left(1-w_{0}\right)-\left(w_{0}+J(v)\right) v\right)\left(w_{0}+v\right)=0 .
$$

Consider first the linear equation

$$
v^{\prime \prime}+c v^{\prime}+\left(J(v)\left(1-w_{0}\right)-w_{0} v\right) w_{0}=0
$$

and integrate it from $N$ to $+\infty$ :

$$
-v^{\prime}(N)-c v(N)+I_{+}(N)=0,
$$

where

$$
I_{+}(N)=w_{0}\left(1-w_{0}\right) \int_{N}^{\infty} d x \int_{-\infty}^{\infty} \phi(x-y) v(y) d y-w_{0}^{2} \int_{N}^{\infty} d x \int_{-\infty}^{\infty} \phi(x-y) v(x) d y .
$$

Since $1-w_{0}>w_{0}$, then by virtue of Lemma $7, I_{+}(N)>0$ for any $N$. We recall that $v(x)$ is positive and decreasing. Therefore, equality (3.14) can take place only if $c>0$.

The corresponding integral remains positive for equation (3.12) since $J(v) \rightarrow 0$ as $x \rightarrow+\infty$ and for any $\epsilon>0, w_{0}+J(v) \leqslant w_{0}+\epsilon, x \geqslant N$ for $N$ sufficiently large. Therefore, integrating (3.12), we obtain, as before, that $c>0$.

Suppose now that there exists a decreasing solution $u(x)$ of equation (2.6) such that $u(x) \rightarrow w_{0}$ as $x \rightarrow-\infty$. Put $v(x)=w_{0}-u(x)$. Then $v(x)$ is a positive increasing function. As above, we obtain equation (3.13). Integrating it from $-\infty$ to $N$, we obtain

$$
v^{\prime}(N)+c v(N)+I_{-}(N)=0,
$$

where

$$
I_{-}(N)=w_{0}\left(1-w_{0}\right) \int_{-\infty}^{N} d x \int_{-\infty}^{\infty} \phi(x-y) v(y) d y-w_{0}^{2} \int_{-\infty}^{N} d x \int_{-\infty}^{\infty} \phi(x-y) v(x) d y .
$$

It follows from (3.11) that $I_{-}(N) \geqslant 0$. Then from (3.15), $c<0$. Thus, we have proved the following lemma. 
Lemma 8. If there exists a decreasing solution of equation (2.6) with the limits $w(-\infty)=w_{-}$, $w(+\infty)=w_{0}$, then $c>0$. If such solution has the limits $w(-\infty)=w_{0}, w(+\infty)=w_{+}$, then $c<0$.

\subsection{A priori estimates of solutions}

In this section we obtain a priori estimates of monotone solutions of equation (2.6) with conditions (1.4). We begin with the lemma which states exponential behaviour of solutions at infinity.

Lemma 9. There exists a constant $\varepsilon>0$ such that the following estimates of monotone solutions $w_{\tau}(x)$ of problem (2.6), (1.4) hold

$$
\left|w_{\tau}(x)-w_{+}\right| \leqslant K e^{-a x}
$$

for such $x$ that $\left|w_{\tau}(x)-w_{+}\right| \leqslant \varepsilon$, and

$$
\left|w_{\tau}(x)-w_{-}\right| \leqslant K e^{-b x}
$$

for such $x$ that $\left|w_{\tau}(x)-w_{-}\right| \leqslant \varepsilon$. Moreover, constants $K>0, a>0$ and $b>0$ are independent of $\tau$ and of solution $w_{\tau}(x)$.

Proof. Consider first behavior of solutions of equation (2.6) as $x \rightarrow \infty$. Since $w_{\tau} \rightarrow w_{+}=0$, and it is monotonically decreasing, then for sufficiently large $x$ we can estimate the integral $J_{\tau}(w)$ by a small constant $\beta>0$ such that $\beta<\alpha$,

$$
J_{\tau}(w(x)) \leqslant \beta, \quad \forall x>x_{0},
$$

which provides

$$
J_{\tau}(w(x)) w(x)(1-w(x))-\alpha w(x) \leqslant(\beta-\alpha) w(x), \quad \forall x>x_{0} .
$$

We can now compare solutions of equation (2.6) with the equation

$$
v^{\prime \prime}+c v^{\prime}+(\beta-\alpha) v=0, \quad \beta<\alpha
$$

Exponential behaviour of solutions of this equation is well known. It remains to prove that monotone solutions $w(x)$ of equation (2.6) can be majorated by solutions $v(x)$ of the previous equation, i.e.

$$
w(x) \leqslant v(x), \quad \forall x>x_{0} .
$$

To do so, consider the difference $z(x)=v(x)-w(x)$. It satisfies the following equation:

$$
z^{\prime \prime}+c z^{\prime}+(\beta-\alpha) z+\left((\beta-\alpha) w-J_{\tau}(w) w(1-w)-\alpha w\right)=0 .
$$

It can be rewritten as

$$
z^{\prime \prime}+c z^{\prime}+(\beta-\alpha) z+g(x)=0
$$


where $g(x) \geqslant 0$ for all $x>x_{0}$. Consider this equation on the half-axis $\left[x_{0},+\infty\right)$ with the boundary conditions $z\left(x_{0}\right)=0$ and $z\left(x_{0}\right) \rightarrow 0$ as $x \rightarrow 0$. If we prove that solutions of this problem satisfy $z(x) \geqslant 0$ for all $x \geqslant x_{0}$, then estimate (3.18) will be proved. Let us assume that this is not true. Hence $z(x)$ has negative values. Since it converges to zero at infinity, then there exists a point $x_{2}>x_{0}$ where the function $z(x)$ attains its negative minimum. At this point we have $z^{\prime \prime}\left(x_{2}\right) \geqslant 0, z^{\prime}\left(x_{2}\right)=0,(\beta-\alpha) z\left(x_{2}\right)>0$ and $g\left(x_{2}\right)>0$. We obtain a contradiction in signs in equation (3.19). Estimate (3.16) follows from (3.18). Estimate (3.17) can be proved in a similar way.

Lemma 10. There exists a constant $\chi>0$ such that outside of the $\varepsilon$-neighbourhoods of the points $w_{+}$and $w_{-}$the following estimate holds $\left|w_{\tau}^{\prime}(x)\right| \geqslant \chi$ for arbitrary monotone solution $w_{\tau}(x)$ of the problem (2.6), (1.4). Constant $\chi$ is independent of the parameter $\tau$ and of solution $w_{\tau}(x)$, constant $\varepsilon$ is defined in Lemma 9.

Proof. Let us assume that the assertion of the lemma does not hold. Then there exist sequences $\left\{\tau_{k}\right\},\left\{w_{\tau_{k}}\right\},\left\{x_{k}\right\}$ such that $w_{\tau_{k}}^{\prime}\left(x_{k}\right) \rightarrow 0$ and points $x_{k}$ do not belong to $\varepsilon$-neighbourhoods of points $w_{-}$and $w_{+}$. We can assume that $\tau_{k} \rightarrow \tau_{0}$ and $c_{k} \rightarrow c_{0}$, where $\tau_{0} \in[0,1], c_{0}$ is a constant, and $c_{k}$ is the speed of the wave $w_{\tau_{k}}$.

Since solutions of equation (2.6) are invariant relative to translation with respect to $x$, we can assume that $\left|w_{\tau_{k}}(0)-w_{-}\right|=\varepsilon$. We can also assume that the sequences $\left\{w_{\tau_{k}}(0)\right\}$ and $\left\{w_{\tau_{k}}^{\prime}(0)\right\}$ are convergent with limits $y$ and $z$, respectively. Denote by $v^{(1)}(x)$ the solution of equation (2.6) for $c=c_{0}, \tau=\tau_{0}$ with initial conditions $v^{(1)}(0)=y, v^{(1) \prime}(0)=z$. It is clear that $v^{(1)}(x) \rightarrow w_{-}$as $x \rightarrow-\infty$.

Let us suppose that $v^{(1)}(x) \rightarrow w_{+}$as $x \rightarrow \infty$. Then, outside of the $\varepsilon$-neighbourhoods of the points $w_{-}, w_{+}$, function $v^{(1)}(x)$ exists on a finite interval with respect to $x$. Since solutions $w_{\tau_{k}}(x)$ converge to $v^{(1)}(x)$, then, for some $x=x_{0}$, we have $v^{(1) \prime}\left(x_{0}\right)=0$, and the point $v^{(1)}\left(x_{0}\right)$ lies outside the $\varepsilon$-neighbourhoods of the points $w_{-}, w_{+}$. This contradicts Lemma 4 . Thus $v^{(1)}(x)$ is a monotone (not necessarily strict) function not tending towards $w_{+}$. Then $v^{(1)}(x)$ tends to $w_{0}$ as $x \rightarrow \infty$, where $w_{0}$ is such that $w_{0}\left(1-w_{0}\right)-\alpha=0$.

In a similar way we may prove existence of function $v^{(2)}(x)$ having limits $w_{0}$ as $x \rightarrow-\infty$ and $w_{+}$as $x \rightarrow \infty$. Thus we have simultaneously two solutions of equation (2.6) with the same wave speed $c=c_{0}$. This contradicts Lemma 8, which completes the proof of the lemma.

Lemma 11. Let $\mathfrak{M}_{r}(r \geqslant 0)$ be the set of all monotone solutions of problem (2.6),(1.4) for all $\tau$ such that for $x_{0}$ defined by

$$
w_{\tau}\left(x_{0}\right)=w_{0}
$$

holds the estimation $\left|x_{0}\right| \leqslant r$. Then there exists such a constant $M_{r}$ that for all $w \in \mathfrak{M}_{r}$ the following estimate holds,

$$
\|w-\psi\|_{C_{\mu}^{2+\alpha}(\mathbb{R})} \leqslant M_{r} .
$$


Proof. Let $w$ be from $\mathfrak{M}_{r}$ and points $x_{1}$ and $x_{2}$ be defined by the equalities

$$
\left|w\left(x_{1}\right)-w_{-}\right|=\varepsilon, \quad\left|w\left(x_{2}\right)-w_{+}\right|=\varepsilon,
$$

where the constant $\varepsilon$ is defined in Lemma $9, \varepsilon<w_{0}$. Then

$$
x_{1}<x_{0}<x_{2}, \quad\left|x_{0}\right| \leqslant r .
$$

Let $\chi$ be the constant in the estimate of $w^{\prime}(x)$, presented in Lemma 10. Then

$$
0<x_{2}-x_{1}<\left(w_{-}-w_{+}\right) / \chi:=N \text {. }
$$

We suppose that $\chi<1$. From (3.21) and (3.22) we obtain that

$$
\left|x_{1}\right| \leqslant N+r, \quad\left|x_{2}\right| \leqslant N+r .
$$

From Lemma 9 we conclude that solutions $w(x)$ approach $\psi(x)$ exponentially outside the interval $\left[x_{1}, x_{2}\right]$. Thus we conclude that

$$
\|w-\psi\|_{C_{\mu}^{2+\alpha}(\mathbb{R})}=\|w-\psi\|_{C_{\mu}^{2+\alpha}\left(\left[x_{1}, x_{2}\right]\right)} .
$$

This norm, in turn, is

$$
\|w-\psi\|_{C_{\mu}^{2+\alpha}\left(\left[x_{1}, x_{2}\right]\right)}=\|(w-\psi) \mu\|_{C^{2+\alpha}\left(\left[x_{1}, x_{2}\right]\right)} .
$$

The estimate of the norm in the right-hand side follows from the estimate of the Hölder norm of the functions $w$ and $\mu$. The former follows from Lemma 6 for the function itself and its first two derivative. The norm $C^{\alpha}$ of the second derivative can be obtained from the equation. The estimate of the Hölder norm of $\mu$ follows from the boundedness of the interval.

Proposition 1. Consider the homotopy defined in Section 2.2 and the equation

$$
w^{\prime \prime}+c w^{\prime}+J_{\tau}(w) w(1-w)-\alpha w=0, \quad(\tau \in[0,1]) .
$$

1) The following estimate holds for all monotone solutions w(x) of problem (2.6), (1.4),

$$
\|w-\psi\|_{C_{\mu}^{2+\alpha}} \leqslant R
$$

where $R>0$ is some constant.

2) For some $r>0$ we have the estimate

$$
\left\|w_{M}-w_{N}\right\|_{C_{\mu}^{2+\alpha}} \geqslant r
$$

where $w_{M}$ and $w_{N}$ are, respectively, an arbitrary monotone and a nonmonotone solution of problem (2.6), (1.4). 
Proof. Up to now we considered $c$ as a constant, now we consider it as a functional $c(u)$ introduced previously, $u(x)=w(x)-\psi(x)$. Let us denote the solution of equation $w(x)=w_{0}$ by $x_{0}$ and prove that there exists a constant $r>0$ such that $\left|x_{0}\right|<r$ for all monotone solutions of our problem and for all $\tau \in[0,1]$. Let us assume the contrary. Then there exists a sequence $\left\{x_{k}\right\}$ such that $\left|x_{k}\right| \rightarrow \infty$ with $k \rightarrow \infty$ and a sequence of solutions $\left\{w_{k}(x)\right\}$ with $c=c\left(u_{k}\right), u_{k}(x)=w_{k}(x)-\psi(x)$ are such that $w_{k}\left(x_{k}\right)=w_{0}$. Let

$$
v_{k}(x)=w_{k}\left(x+x_{k}\right)-\psi(x),
$$

so that $v_{k}+\psi \in \mathfrak{M}_{0}$ (see Lemma 11), and, thus, we have the estimate

$$
\left\|v_{k}\right\|_{C_{\mu}^{2+\alpha}} \leqslant M_{0}
$$

From functionalisation of parameter $c$ we have

$$
\rho(u)=\left(\int_{R}\left(u(x)+\psi(x)-w^{+}\right)^{2} \sigma(x) d x\right)^{1 / 2},
$$

which provides

$$
\rho\left(u_{k}\right)=\left(\int_{R}\left(v_{k}(x)+\psi(x)-w^{+}\right)^{2} \sigma\left(x+x_{k}\right) d x\right)^{1 / 2} .
$$

Further we show that $c\left(u_{k}\right)=\ln \rho\left(u_{k}\right)$ is unbounded, which leads to a contradiction with the a priori estimates of the speed obtained in Section 3.3. When $x_{k} \rightarrow \infty, \sigma\left(x+x_{k}\right) \rightarrow 1$ and $\left|v_{k}\right| \rightarrow 0$. Function $\psi(x)$ is different from $w_{+}$when $-x$ is big, thus $\rho\left(x_{k}\right) \rightarrow \infty$ as $x_{k} \rightarrow \infty$. We should consider as well the case when $x_{k} \rightarrow-\infty$. From the definition of the norm in Hölder spaces we obtain that $\left|v_{k}(x)\right| \leqslant N(\mu(x))^{1 / 2}$. Thus there exists square summarible function $y(x)$ such that $\left|v_{k}(x)\right|<y(x)$. This allows the conclusion that $\rho\left(u_{k}\right) \rightarrow 0$ as $x_{k} \rightarrow-\infty$.

Thus we have shown that all monotone solutions of problem (2.6),(1.4), in which $c=c(u)$, belong to $\mathfrak{M}_{r}$ for some $r>0$ and validity of the first part of the proposition follows from Lemma 11 .

Let us now prove the second part of the proposition. Introduce the notation,

$$
u_{M}=w_{M}-\psi, \quad u_{N}=w_{N}+\psi .
$$

Assume that the assertion of the second part of the proposition does not hold. We can then find sequences of solutions of equation (2.6) $\left\{u_{M}^{(k)}\right\}$ and $\left\{u_{N}^{(k)}\right\}$ such that

$$
\left\|u_{M}^{(k)}-u_{N}^{(k)}\right\|_{C_{\mu}^{2+\alpha}} \underset{k \rightarrow \infty}{\longrightarrow} 0 .
$$

Since all the functions $u_{M}^{(k)}$ are in the ball of radius $R$ of the space $C_{\mu}^{2+\alpha}(\mathbb{R})$, we obtain the strong convergence of $\left\{u_{M}^{(k)}\right\}$ to some $u_{M}^{(0)}$,

$$
\left\|u_{M}^{(k)}-u_{M}^{(0)}\right\|_{C^{2}} \underset{k \rightarrow \infty}{\longrightarrow} 0
$$

We let $w^{(0)}=u_{M}^{(0)}+\psi$. Then function $w^{(0)}$ is a solution of problem (2.6),(1.4) for some $\tau$ and $c$ and

$$
\left\|w_{M}^{(k)}-w^{(0)}\right\|_{C^{2}} \underset{k \rightarrow \infty}{\longrightarrow} 0
$$


Then from (3.23) and (3.24) we have

$$
\left\|w_{N}^{(k)}-w^{(0)}\right\|_{C^{2}} \underset{k \rightarrow \infty}{\longrightarrow} 0
$$

Applying Lemma 5 to sequence $\left\{w_{N}^{(k)}\right\}$ we obtain a contradiction, which completes the proof of the proposition.

Consider a ball $\|u\|_{C_{\mu}^{2+\alpha}} \leqslant R$ of space $C_{\mu}^{2+\alpha}(\mathbb{R})$, in which all solutions of equation (2.6) are contained. We set $R_{1}=R+1$ and select $r, 0<r<1$, such that for solutions of equation (2.6) in the ball $\|u\|_{C_{\mu}^{2+\alpha}} \leqslant R_{1}$ we have the inequality

$$
\left\|w_{M}-w_{N}\right\|_{C_{\mu}^{2+\alpha}} \geqslant r
$$

In Theorem 2 we have proved that operator $A_{\tau}(u)$ is proper with respect to both $\tau$ and $u$. This means that the set of solutions of equation $A_{\tau}(u)=0$ is compact in the ball $\|u\|_{C_{\mu}^{2+\alpha}} \leqslant R_{1}$. For a fixed solution $u_{M}(x)$ we construct the ball $K\left(u_{M}\right)$ of radius $r$ and center at the point $u_{M}$. By virtue of compactness of the set of solutions $u_{M}$, we can select from a covering of this set by balls $K\left(u_{M}\right)$ a finite subcovering. We denote by $G_{k}, k=1, \ldots, N$, the set of domains formed by the union of the balls from this subcovering, $\Gamma_{k}$ are boundaries of these domains.

It is obvious that any $u_{N} \notin \bigcup_{k=1}^{N}\left[G_{k}+\Gamma_{k}\right]$ and all solutions $u_{M}$ belong to $\bigcup_{k=1}^{N} G_{k}$ for all $\tau \in[0,1]$. We thus have separated all monotone solutions from all nonmonotone solutions, which allows us to prove the main result of this work stated in Theorem 1.

Proof. Consider the homotopy (2.4)-(2.6). As it is shown in Section 2, the topological degree can be introduced for the operator $A_{\tau}$. By virtue of a priori estimates obtained in this section we can use the Leray-Schauder method. As shown in [2], equation (2.6) with conditions (1.4) has a solution in the form of monotone travelling wave when $\tau=0$. This solution is unique up to translation in space. Functionalization of the wave speed removes the zero eigenvalue of the linearized operator. Therefore, the spectrum of the linearized operator lies completely in the left-half plane. Thus topological degree for the operator $A_{\tau}(u)$, introduced in (2.3), equals 1 for $\tau=0$. Consequently, the topological degree for operator $A_{1}$, corresponding to the initial equation (1.3) also equals 1 , whence the existence of a solution follows.

\section{References}

[1] S. Ai. Traveling wave fronts for generalized Fisher equations with spatio-temporal delays. J. Differential Equations, 232 (2007), 104-133.

[2] A. Apreutesei, A. Ducrot, V. Volpert. Competition of species with intra-specific competition. Math. Model. Nat. Phenom., 3 (2008), 1-27.

[3] N. Apreutesei, A. Ducrot, V. Volpert. Travelling waves for integro-differential equations in population dynamics. Discrete Cont. Dyn. Syst. Ser. B, 11 (2009), 541-561. 
[4] N.F. Britton. Spatial structures and periodic travelling waves in an integro-differential reaction-diffusion population model. SIAM J. Appl. Math., 6 (1990), 1663-1688.

[5] A. Ducrot, Travelling wave solutions for a scalar age-structured equation, Discrete Contin. Dyn. Syst. Ser. B , 7 (2007), 251-273.

[6] P. C. Fife, J. B. McLeod. The approach of solutions of nonlinear diffusion equations to travelling wave solutions. Bull. Amer. Math. Soc., 81 (1975), 1076-1078.

[7] A. Friedman. Partial differential equations of parabolic type. Prentice-Hall, Englewood Cliffs, 1964.

[8] S. Génieys, V. Volpert, P. Auger. Pattern and waves for a model in population dynamics with nonlocal consumption of resources. Math. Model. Nat. Phenom., 1 (2006), 63-80.

[9] S. A. Gourley. Travelling front solutions of a nonlocal Fisher equation. J. Math. Biol. 41 (2000), 272-284.

[10] Ya. I. Kanel. The behavior of solutions of the Cauchy problem when the time tends to infinity, in the case of quasilinear equations arising in the theory of combustion. Soviet Math. Dokl. 1 (1960), 533-536.

[11] R. Lefever, O. Lejeune. On the origin of tiger bush. Bul. Math. Biol., 59 (1997), No. 2, 263-294.

[12] A.I. Volpert, V.A. Volpert. Applications of the rotation theory of vector fields to the study of wave solutions of parabolic equations. Trans. Moscow Math. Soc., 52 (1990), 59-108.

[13] A. Volpert, Vl. Volpert, Vit. Volpert. Travelling wave solutions of parabolic systems. 1994, AMS, Providence.

[14] V. Volpert, A. Volpert, J.F. Collet. Topological degree for elliptic operators in unbounded cylinders. Adv. Diff. Eq., 4 (1999), 777-812.

[15] V. Volpert, A. Volpert. Properness and topological degree for general elliptic operators. Abstract and Applied Analysis, 2003 (2003), 129-181.

[16] A. Volpert, V. Volpert. Normal solvability of general linear elliptic problems. Abstract and Applied Analysis, 7 (2005), 733-756.

[17] Y. Wang, J. Yin, Travelling waves for a biological reaction diffusion model with spatio- temporal delay, J. Math. Anal. Appl., 325 (2007), 1400-1409.

[18] Z.C. Wang, W.T. Li, S. Ruan, Travelling wave fronts in reaction-diffusion systems with spatio-temporal delays, J. Diff. Equations, 222 (2006), 185-232. 